\title{
Erratum zu: Urbane Mobilität als Schlüssel für eine neue Gesellschaft
}

\section{T. Ambs, K. Pipahl, Urbane Mobilität als Schlüssel für eine neue Gesellschaft, https://doi.org/10.1007/978-3-658-30729-5}

Liebe Leserin, lieber Leser, vielen Dank für Ihr Interesse an diesem Buch. Leider haben sich trotz sorgfältiger Prüfung Fehler eingeschlichen, die uns erst nach Drucklegung aufgefallen sind. Die nachfolgenden Korrekturen wurden jetzt ausgeführt.

- Die Namen der Gastautoren wurden im Inhaltsverzeichnis ergänzt.

- Die Beiträge der Gastautoren wurden um die Auflistung der entsprechenden Literatur ergänzt.

- Es wurden kleinere Korrekturen am Text vorgenommen.

Die aktualisierte Version des Buches finden Sie unter https://doi.org/10.1007/978-3-658-30729-5

(C) Springer Fachmedien Wiesbaden GmbH, ein Teil von Springer Nature 2021 E1 T. Ambs, K. Pipahl, Urbane Mobilität als Schlüssel für eine neue Gesellschaft, https://doi.org/10.1007/978-3-658-30729-5_5 